\title{
UBIQUITOUS COMPUTING: OBJECT TRACKING AND MONITORING IN CONSTRUCTION PROCESSES UTILIZING ZIGBEETM NETWORKS
}

\author{
Mirosław J. Skibniewski \\ A.J. Clark Chair Professor \\ Dept. of Civil and Environmental Engineering \\ UNIVERSITY OF MARYLAND \\ 1188 Glenn L. Martin Hall \\ College Park, MD 20742-3021, USA \\ mirek@umd.edu
}

\author{
Won-Suk Jang \\ Ph.D. Candidate \\ Dept. of Civil and Environmental Engineering \\ UNIVERSITY OF MARYLAND \\ 0154 Glenn L. Martin Hall \\ College Park, MD 20742-3021, USA \\ wsjang@umd.edu
}

\begin{abstract}
A large-scale of civil infrastructure systems is associated with great amounts of project resources and activities interoperated with various participants and organizations. Well-defined methodology for information acquisition and communication became more critical in the management of civil infrastructure than ever before. Rapidly emerging wireless sensor technology has been drawing a log of attention as a possible method to realize the ubiquitous computing environment in everyday life, and more advanced strategy for implementing ubiquitous interfaces between device and networks needs to be investigated in the application of civil infrastructure. This paper introduces the conceptual understanding and strategy of ZigBee networks to be deployed in the civil infrastructure systems. In addition, the prototype application for object tracking and monitoring system in construction site using ZigBee networks is proposed to provide an insight for the industrial practices in sensor- and network-based ubiquitous computing.
\end{abstract}

Keywords: Ubiquitous computing, ZigBee sensor network, civil infrastructure, localization systems

\section{INTRODUCTION}

Rapidly growing wireless sensor technology has been drawing a lot of attention as a possible method to realize the ubiquitous computing environment in everyday life. Under the ubiquitous environment, numerous sensors and communication devices can be utilized for connection throughout complicated networks, capable of monitoring and detecting the physical events. Industrial applications can benefit from the emergence of ubiquitous computing in building automation, environmental monitoring, and surveillance of civil infrastructure with improved efficiency and effectiveness. Particularly, a large-scale of civil infrastructure is associated with great amounts of project resources and activities interoperated with various participants and organizations. In consequence, diverse roles and functionalities among project events and information process need to be improved, and more advanced strategy for implementing ubiquitous interfaces between device and networks needs to be investigated in the application of civil infrastructure systems.

Ubiquitous computing is an emerging concept in computing, which integrates computation capabilities into the physical environment rather than being perceived as a visible object. Hundreds of sensor and communication devices are embedded into physical life space, and they detect and monitor the physical activities in the place of human's eyes and ears. The sensor networks are located to everywhere to perform an ad hoc arrangement that offers a higher degree of flexibility for settings and increased number of sensor nodes. However, the requirements and specifications of sensor network infrastructure for ubiquitous computing differ from various applications: from home automation to the surveillance of large-scale civil infrastructure systems. In addition, feasible methodologies and frameworks focused on the civil engineering industry - combined with advanced information technology - have not yet taken a concrete shape due to the lack of cooperation among different participants, lack of information sharing, and inefficient use of emerging technologies.

As one of the global networking specification for wireless connectivity in the implementation of ubiquitous computing environment, ZigBee ${ }^{\mathrm{TM}}$ emerges as a new industrial standard for ad hoc networks based on IEEE specification [11]. Aimed at control and sensor applications, ZigBee is a superset of the IEEE 802.15.4 and promises the robust and reliable, and self-configuring networks that provide a simple, cost-effective and batteryefficient application. The ZigBee Alliance released its specification to the public in June 2005, and they have focused on providing a technology to take advantage of short-range wireless protocol, flexible mesh networking, strong security tools, well-defined application frameworks, and a complete interoperability. It is believed that the evolving information technology, together with the ZigBee standard, is able to envision the new paradigm of civil engineering applications focusing on home control, building automation and industrial control and monitoring.

In this paper, technological details of the ZigBee networking protocol will be discussed. Network topology and beaconing scheme to formulate the ubiquitous computing environment will be investigated for the anticipated framework of redesign and reconfiguration of current legacy systems for the deployment of emerging sensor network technologies. Finally, prototype application deployed for object tracking and a monitoring system on a construction job site within the ZigBee network is illustrated to present the insight for the industrial practices in sensor-based ubiquitous computing. 


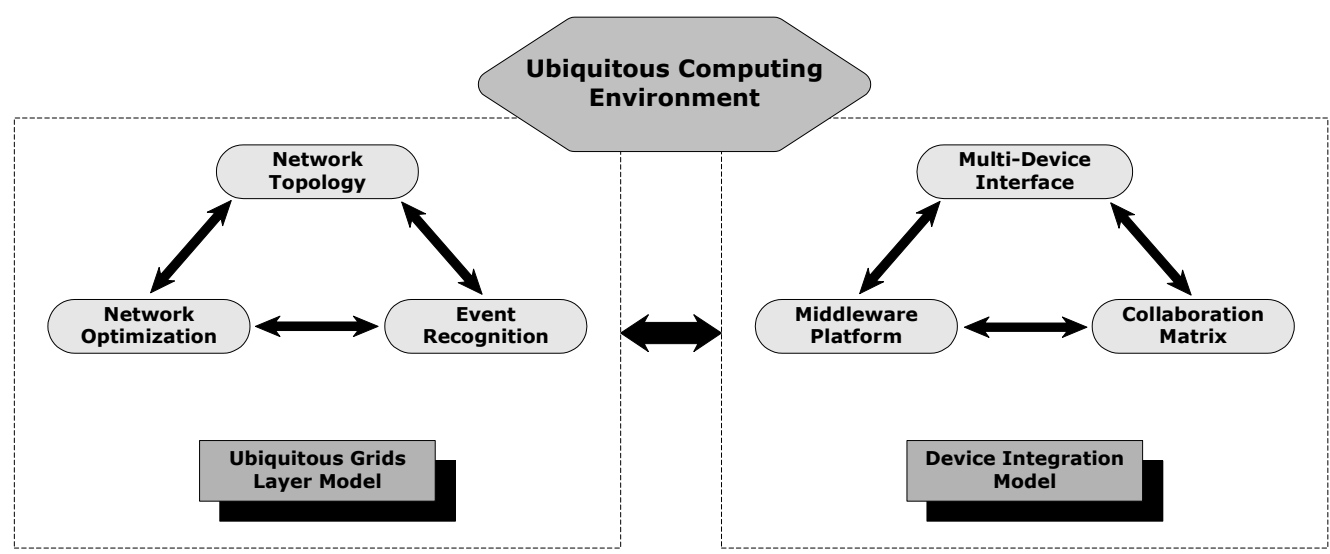

Figure 1: Framework modeling of ubiquitous computing for civil infrastructure systems [12].

\section{RELATED WORK}

A shift to ubiquitous computing is under way in civil infrastructure for project management and surveillance applications. With the emerging technology of advanced sensors and networking, an opportunity emerges for civil engineers to implement ubiquitous computing, promising greater effectiveness, automation, and improvement in project tasks. Jang [12] proposed a framework modeling of ubiquitous computing for civil infrastructure systems that consists of ubiquitous grids layer model and device integration model, which is shown in Figure 1. This research discussed the communication services with multidevice and distributed user-groups in civil infrastructure domains. In addition, the critical components of ubiquitous computing model, such as network topology analysis, event recognition and collaboration matrix, were introduced to address the implementation of sensor- and network-based computing environment in civil infrastructure.

Sensor network interface provides a critical functionality in acquiring and communicating the information among hundreds of distributed entities involved in the civil infrastructure systems. For the research activities of the development of sensor and networking platform applicable to civil infrastructure, Sazonov [17] presented a design of wireless intelligent sensor and actuator network (WISAN) for the applications in structural health monitoring. WISAN addresses the issues of achieving a low cost per sensor, higher reliability, sources of energy for the network nodes and energyefficient distribution of the computational load. Several researchers [2] and [13] focused on embedded sensing systems to capture the project history and monitoring data in terms of wireless communication applicable to civil infrastructure. However, such researchers were faced with a challenge of providing wireless networking topology and data transmission protocol for the wireless communication method in large and complex civil infrastructure environment.

Since the release of ZigBee specification in 2005 [11], there has been an increased interest in building automation and industrial monitoring using ZigBee standard. Low cost, low power and networking flexibility has provided a potential to realize the wireless network environment for ubiquitous computing. Possible scenarios of foreseen application using ZigBee were presented for blueprint of a ubiquitous computing environment, and several feasibility and reliability analyses have been studied [1], [4], [6], [7], [8], and [10] to suggest the implementation strategy of ZigBee technology, comparing with several traditional technologies of wireless networks. Since ZigBee technology is still in the premature stage to address robust and reliable communication methods, significant effort on R\&D in both research institutes and industrial organizations has been put to provide the better solution of mesh network, applications profiles, device descriptions and interoperability.

Location tracking algorithm [3], presented later in this paper, has fostered a growing interest in mobile systems implementing ubiquitous computing environments. The majority of existing localization methods leverages the existence of IEEE 802.11 base stations which transmit radio power of $100 \mathrm{mV}$ per base station. Such radios are classified differently with the low power IEEE 802.15.4 compliant radios [16]. However, most industrial applications for wireless sensing require the platform that has the low cost and low power consumption to be deployed in a large scale geographical area. Even though several researches has been focused on location awareness techniques based on signal strength of Bluetooth network [5] and[14], which provides low cost and low power in signal transmission, Bluetooth has a limitation relevant to construction sites due to very short coverage range of 30 feet.

In general, signal strength based localization can be divided into map-based [3], [15] and distance-based [9], [18] algorithms. In the map-based algorithms, a large set of sample points that cover the whole geographical area collect one or more signal strength measurements from all visible beacon nodes. Each sample point is then mapped into either signal strength vector or a signal strength probability distribution. Despite the map-based localization methods provide relatively small location errors, they have some limitations because they require an excessive amount of profiling processes. On the other hand, distance-based localization algorithms utilize the distance prediction model either by triangulation or 
trilateration method because the raw signal strength data can be mapped into distance through a signal propagation model. The measured distance can be either used directly in order to determine the location of mobile devices or used in a probabilistic localization algorithm.

Although advanced sensing and networking technologies promise a great advantage of deployment in construction or civil infrastructure applications, little research have been pursued to provide a solid framework and reasoning for exploitation of object tracking system in those areas. In combination of signal strength based localization algorithm and ZigBee networking capability with low power and multi hoc feature can present the opportunity in tracking and monitoring the wide variety of components for construction work process and material tracking system, enabling to reduce the construction project cost and large amount of labor.

\section{ZIGBEE NETWORKS}

Conventional networking system with wired sensor is quite different from the wireless sensor network in that existing sensor networks were configured with a small number of sensor points due to constraints of installation cost. Because of this limitation, wired sensor networks utilized the sensor points into small number of points in measurement considered as the important representation of information, therefore, individual observational data was quite important in controlling or monitoring the civil infrastructure. On the other hand, the capability of acquiring the measurement in wireless sensor networks is more flexible and expandable for configurations and an increased number of sensor points by establishing ad hoc networks. Therefore, wider scope of deployment strategy can be applied in terms of ubiquitous computing networks used by wireless sensor network, and large number of information can be described to be important in the mechanism of data collection and acquisition.

In general, sensors and communication devices used for the deployment to ubiquitous computing in civil infrastructure systems are not required to have high-speed communication capacities; rather more consideration needs to be focused on a limited amount of delay in communication and a low energy consumption. In this perspective, ZigBee is emerging network technology as a wireless communication standard capable of realizing the ubiquitous environment to satisfy such requirements. ZigBee is the product of the ZigBee Alliance, an organization of manufacturers dedicated to developing a new networking technology, and it is aimed to industrial and home application into wireless connectivity. The ZigBee specification has been released to the public in June 2005, and products supporting the ZigBee standard are now announcing in the market. The ZigBee specification takes advantage of the IEEE 802.15.4 wireless protocols as basic communications method, and expands on this with a robust mesh network, application profiles, interoperability and device description. The promise of ZigBee application can be found in robust and reliable, self-configuring and self-healing networks that provide a simple, cost-effective and battery-efficient approach to add wireless to mobile and fixed communication devices.

\subsection{IEEE 802.15.4 and ZigBee Structure}

The IEEE 802.15.4, released in May 2003, was selected by ZigBee Alliance [11] as a lower-rate version of the IEEE 802.15 series standard for wireless communications of the Wireless Personal Area Networks (WPAN). The IEEE 802.15.4 standard defined the RF capacity with the system operating in three license-free bands for global communication, and RF link parameters are shown in the Table 1.

Table 1: Frequency allocations and physical layer in IEEE 802.15.4 [1]

\begin{tabular}{c|c|c|c}
\hline $\begin{array}{c}\text { Frequency } \\
\text { band }\end{array}$ & $2.4 \mathrm{GHz}$ & $915 \mathrm{MHz}$ & $868 \mathrm{MHz}$ \\
\hline $\begin{array}{c}\text { Number of } \\
\text { channels }\end{array}$ & 16 & 10 & 1 \\
\hline $\begin{array}{c}\text { Bandwidth } \\
\text { (kHz) }\end{array}$ & 5,000 & 2,000 & 600 \\
\hline $\begin{array}{c}\text { Data rate } \\
\text { (kbps) }\end{array}$ & 250 & 40 & 20 \\
\hline $\begin{array}{c}\text { Symbol rate } \\
\text { (ksps) }\end{array}$ & 62.5 & 40 & 20 \\
\hline $\begin{array}{c}\text { Modulation } \\
\text { method }\end{array}$ & O-QPSK & BPSK & BPSK \\
\hline $\begin{array}{c}\text { Diffusion } \\
\text { method }\end{array}$ & DSSS** & DSSS & DSSS \\
\hline $\begin{array}{c}\text { Available } \\
\text { regions }\end{array}$ & Worldwide & USA & Europe \\
\hline
\end{tabular}

O-QPSK $^{*}$ (Offset Quadrature Phase Shift Keying)

$\operatorname{BPSK}^{* *}$ (Binary Phase Shift Keying)

$\operatorname{DSSS}^{* * *}$ (Direct Sequence Spread Spectrum)

The physical layer of the IEEE 802.15.4 has functional capabilities, such as measurement of reception power and notification for link quality and Carrier Sense Multiple Access with Collision Avoidance (CSMA-CA). A radio with data to transmit first seeks to the channel and transmits its packet when the channel is clear. On the other hand, if the channel is occupied or interfered by either 802.15.4 or non-802.15.4 station, the radio holds off from the channel for a certain period of time before again checking the channel for occupancy. In this manner, it is possible to conduct high-quality communications in a platform that is shared with other systems using same frequency band. At Medium Access Control (MAC) layer, IEEE 802.15.4 controls access to the radio channel using CSMA-CA mechanism. Network beacons bind a superframe, allowed by IEEE 802.15.4, and divide it into 16 equally segmented slots to synchronize the attached devices, to identify the personal area network (PAN), and to describe the superframe structure. Hence, the slotted CSMA-CA provides communication method to any device with which other devices will compete within the networks. 


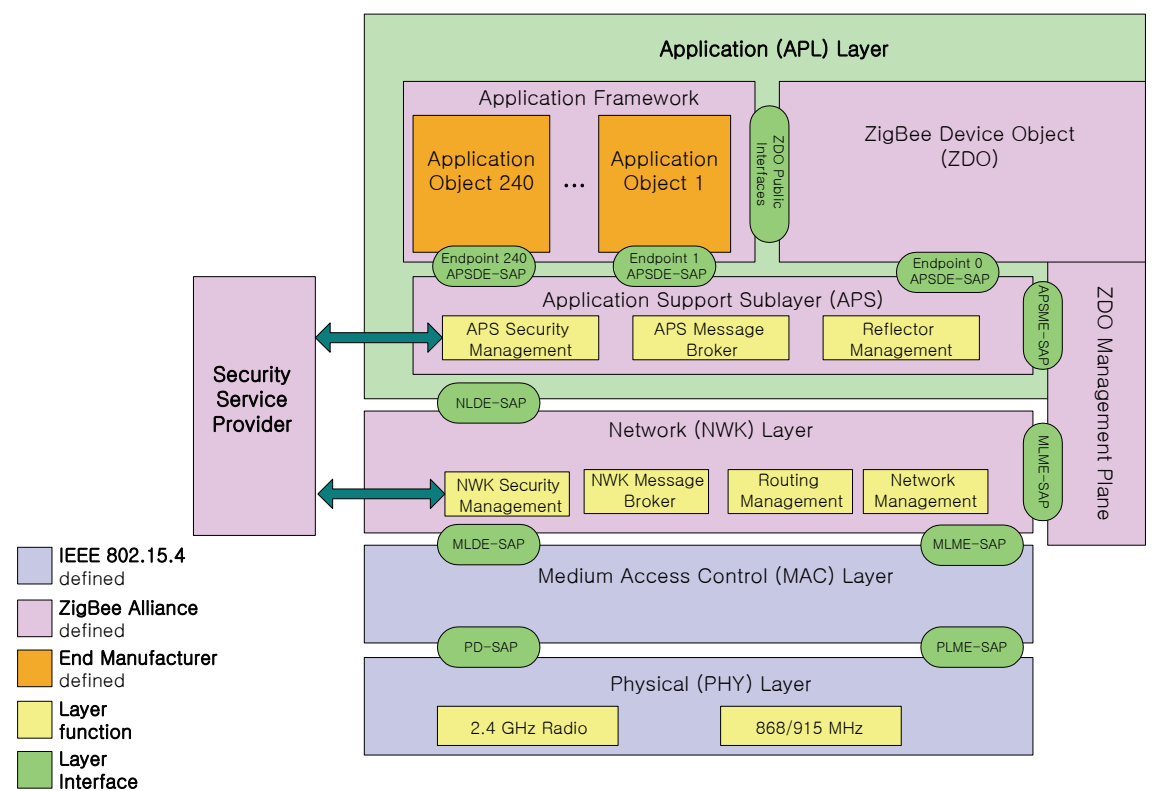

Figure 2: ZigBee stack architecture [11].

The Figure 2 shows the relative organization of the IEEE radio with respect to the ZigBee functionality. On top of the basic IEEE 802.15.4 standard, ZigBee defines the upper layers of the system. The physical and MAC layers take full advantage of the physical radio specified by IEEE 802.15 .4 , which describes a peer-topeer radio using Direct Sequence Spread Spectrum (DSSS). The data rates, channelization, and modulation techniques are employed in those layers. In the logical network, security, and application software layer, the ZigBee networking stack creates the mesh networking capability. Each microcontroller/RF chip combination requires its own ZigBee stack due to the differences in microcontrollers and RF chips, and the ZigBee stack provided by the chip vendor is included with either the microcontroller or RF chip. The application layer is defined by two types of profiles: public profiles are certified by ZigBee alliance for interoperability purpose, and private profiles are for use in closed systems.

\subsection{Zigbee Network Layer}

ZigBee is intended to support three network topologies: star, mesh, and hybrid networks. The star network is very common, and provides the simple configuration of topology. However, when the size of network is large and the network becomes complex, the star network may not take advantage to be used. Mesh network (or peer-to-peer) networks provides high degree of reliability when the signal packets are routed through a variety of nodes. This feature of mesh network can take advantage especially when clear lines of sight between nodes are required due to obstacles that block the radio signals. Mesh networks also make the communication possible to pass through any set of nodes between the sender and receiver. This capability makes the mesh network self-healing and self-configuring even when a node breaks down and is out of service. Finally, hybrid network is essentially a combination of star and mesh networks which is usually deployed to address the most complex network requirements.

A ZigBee network, shown in Figure 3, consists of ZigBee coordinators, ZigBee routers and ZigBee end devices. The coordinator and routers are able to form a star network configuration using PAN coordinator functions, and it is possible to form a multi-hop network by simultaneously configuring the mesh network between the coordinator and routers. On the other hand, the end devices take part in the network communication by linking to the coordinator and routers through star-link networks. The end devices conduct multi-hop communications via connected routers to communicate with other devices connected to the networks.

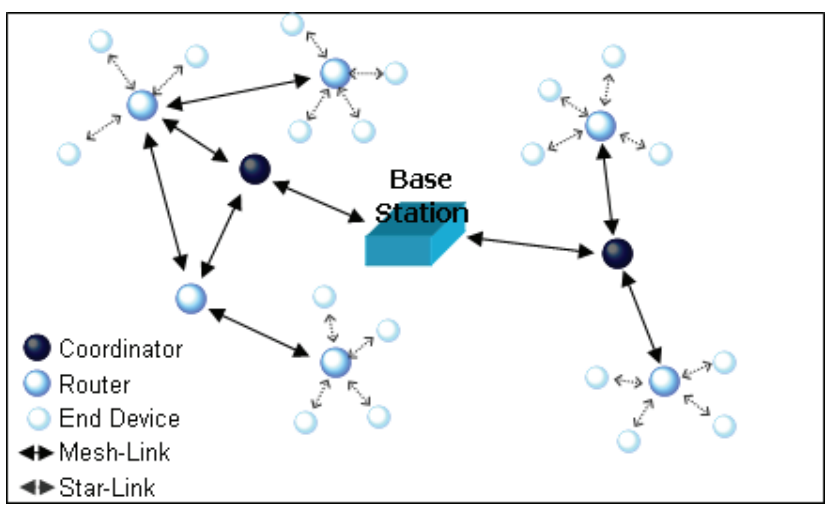

Figure 3: ZigBee Network [11].

\section{PROTOTYPE APPLICATION: OBJECT TRACKING AND MONITORING ON A CONSTRUCTION SITE}

Dynamic nature of the construction supply chains often fails to deliver, locate, track and install the supplies in timely manner, causing project delay and increased cost. Although some of the intangible components such as 
ready-mixed concrete, etc. are not easy to identify as a physical object, the challenging but possible tasks can be identified in many of the prefabricated components delivered, stocked and installed during construction process. Some of the researches focused on material tracking system in construction job site have been presented using decent technologies such as RFID or active/passive badge to track and locate the supply chains, but the difficulties in practical implementation can be found in data sensing and acquisition method due to limited range of coverage and randomly configured obstacles that block the radio signal.

In the prototype application presented in this paper, we develop a new framework deployed by the ZigBee network using smart tags to identify the individual entity of supply chain to be tracked and located in the construction job site. Unlike GPS, currently used in location tracking and navigation, radio signal in ZigBee cannot directly determine the distance between reference point and object's location since the router as a reference point receives only certain level of signal strength from the object within the signal coverage. In consequence, the analysis of signal strength (SS) provides a way of inferring the object's location, and the object's location can be determined by best matching the observed signal strength data based on a set of signal strength measurements at each reference point.

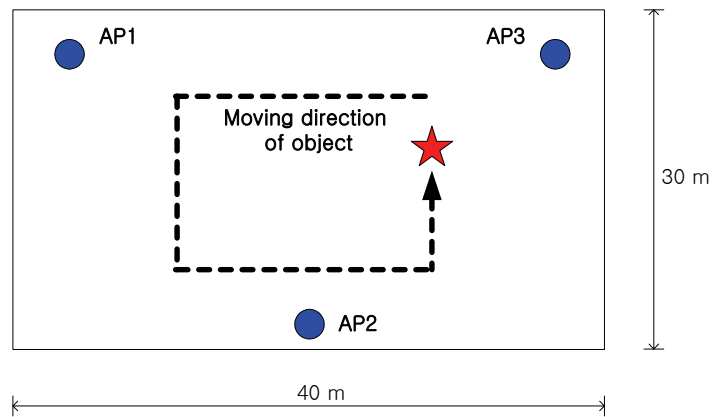

Figure 4: Experimental testbed for tracking the location of moving object

Experimental testbed and procedure are shown in Figure 4 , where the layout of the testbed $(40 \mathrm{~m}$ by $30 \mathrm{~m}$ ) is representing a scaled-down construction job site. Planned procedure for the experiment can be described as follows: three access points equipped with ZigBee chipset are placed at AP1, AP2 and AP3, and mobile device, equipped with IEEE 802.15.4 chipset, is attached to the object. While the mobile object is moving along with the indicated path, the access points receive the variation of SS and signal-to-noise ratio (SNR).

Our approach to determine the object's location is based on nearest neighbors in signal space (NNSS) using trilateration method. Trilateration uses the known locations of two or more reference points, and the measured distance between the object and each reference point. After experimental test to measure hundreds of samples of SS and SNR data are conducted, predicted signal strength and variation of estimated error, in terms of the distance from the location of reference and object, are derived by probabilistic approach as a signal propagation model. Time-segment analysis of measuring the discrete signal strength can also provide the direction vector of the mobile object, which is capable of predicting the moving direction within the network, shown in Figure 5.

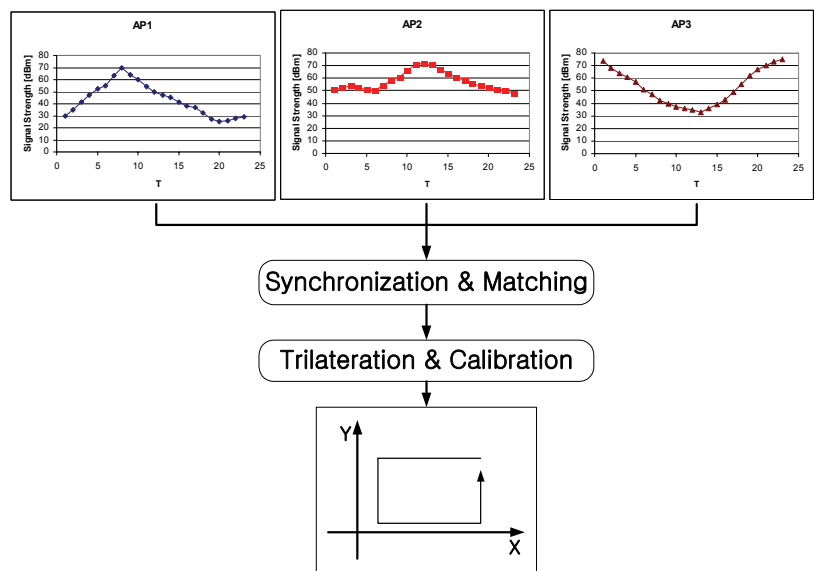

Figure 5: Algorithm procedures for tracking the location of an object.

\section{CONCLUSION AND FUTURE WORK}

This paper describes the proposed ZigBee networking protocol and presents its potential deployment to construction job sites and civil infrastructure systems. An example prototype application in object tracking and monitoring on a construction site utilizing a ZigBee network is illustrated. This example allows a limited insight into a possible future industrial practice using sensor-based ubiquitous computing. A conceptual algorithm for localization of a mobile device on a construction project site has been proposed with the use of a ZigBee network.

Based on the associated components of network specifications and localization described in the paper, we plan to continue our investigation related to the development of advanced algorithm for localization and ad hoc networking strategy, which will provide a framework for the communication in the industrial environment of large scale sensor nodes. As the continuous research activities relating to ubiquitous computing in civil infrastructure systems, the interface design that includes multi-communication protocols, middleware platform, and network topology formulation will be created for practical implementation of a deviceindependent platform in the future.

\section{REFERENCES}

[1] Adams, J. T. (2005), “ZigBee Wireless Technology and the IEEE 802.15.4 Radio - Enabling Simple Wireless," Texas Wireless Symposium 2005, University of Texas, October 26-28, 2005.

[2] Akinci, B. (2004), "Using Sensor Systems and Standard Project Models to Capture and Model Project History for Building Commissioning and 
Facility Management," Facility Area Network Workshop, Civil Engineering Research Lab, UrbanaChampaign, IL, Feb. 26-27, 2004.

[3] Bahal, P. and Padmanabhan, V. (2000), "RADAR: An In-Building RF-based User Location and Tracking System," Proceedings of IEEE INFOCOM, Tel-Aviv, Israel, March 2000.

[4] Baker, N. (2005), "ZigBee and Bluetooth Strength and Weakness for Industrial Application," IEEE Computing \& Constrol Engineering Journal, Vol. 16, No. 2, April/May 2005, pp. 20-25.

[5] Bandara, U., Hasegawa, M., Inoue, M., Morikawa, H. and Aoyama, T. (2004), "Design and Implementation of a Bluetooth Signal Strength Based Location Sensing System," Proceedings of IEEE Radio and Wireless Conference (RAWCON '04), Atlanta, GA, September 19-22, 2004, pp. 319-322.

[6] Cox, D., Jovanov, E. and Milenkovic, A. (2005), "Time Synchronization for ZigBee Networks," Proceedings of the Thrity-Seventh Southeastern Symposium (SSST '05), System Theory, Tuskegee, AL, March 20-22, 2005, pp. 135-138.

[7] Ding, G. and Sahinoglu, Z. (2005), "Reliable Broadcast in ZigBee Networks," Proceedings of IEEE SECON 2005, Sensor and Ad Hoc Communications and Networks, Santa Clara, CA, September 26-29. 2005, pp. 510-520.

[8] Egan, D. (2005), "The Emergence of ZigBee in Building Automation and Industrial Control," IEEE Computing \& Control Engineering Journal, Vol. 16, No. 2, April/May 2005, pp. 14-19.

[9] Elnahrawy, E., Li, X. and Martin, R. M. (2004), "The Limits of Localization Using Signal Strength: A Comparative Study," Proceedings of Sensor and Ad-Hoc Communications and Networks Conference (SECON '04), Santa Clara, CA, October 2004.

[10] Geer, D. (2005), "Users Make a Beeline for ZigBee Sensor Technology," IEEE Computer Society, Computer, Vol. 38, No. 12, December 2005, pp. 1619.

[11] http://www.zigbee.org

[12] Jang, W-. S. and Skibniewski, M. (2006), "Strategy for Applying Ubiquitous Computing and Sensor Networks to Surveillance of Civil Infrastructure Systems," Proceedings of the 8th International Scientific Conference on Computer Aided Engineering, Polanica, Poland, June 21-24.

[13] Krüger, M. and Grosse, C. (2004), "Structural Health Monitoring with Wireless Sensor Networks," OttoGraf Journal, Vol. 15, pp. 77-89.

[14] Li, C. F. and Hwang, R. H. (2005), "A LocationAware P2P Information Sharing System in Bluetooth-based Mobile Ad Hoc Network," International Conference on Wireless Networks, Communications and Mobile Computing (WirelessCom '05), Vol. 2, June 13-16, 2005, Mauri, Hawaii, pp. 1011-1016.

[15] Lorincz, K. and Welsh, M. (2005), "Motetrack: A Robust, Decentralized Aproachto RF-based Location
Tracking," Proceedings of the International Workshop on Location- and Context-Awareness (LoCA '05), Munich, Germany, May 12-13, 2005.

[16] Lymberopoulos, D., Linsey, Q. and Savvides, A. (2006), "An Empirical Characterization of Radio Signal Strength Variability in 3-D IEEE 802.15.4 Networks Using Monopole Antennas," European Workshop on Wireless Sensor Networks (EWSN '06), Zurich, Switzerland, February 13-15, 2006.

[17] Sazonov, E., Jonoyan, K. and Jha, R. (2004), "Wireless Intelligent Sensor Network for Autonomous Structural Health Monitoring," Proceedings of SPIE's $11^{\text {th }}$ Annual International Symposium on Smart Structures and Materials, March 14-18, 2004, San Diego, CA, pp. 5384-5394.

[18] Yedavalli, K., Krishnamachari, B., Ravula, S. and Srinivasan, B. (2005), "Ecolocation: A Technique for RF Based Localization in Wireless Sensor Networks," Proceedings of Information Processing in Sensor Networks (IPSN '05), Los Angeles, CA, April 2005. 